\title{
Cooperative Energy Harvesting Communications with Relaying and Energy Sharing
}

\author{
Kaya Tutuncuoglu and Aylin Yener \\ Department of Electrical Engineering \\ The Pennsylvania State University, University Park, PA \\ Email:kaya@psu.edu, yener@engr.psu.edu
}

\begin{abstract}
This paper considers two-hop communication networks where the transmitters harvest their energy in an intermittent fashion. In this network, communication is carried out by signal cooperation, i.e., relaying. Additionally, the transmitters have the option of transferring energy to one another, i.e., energy cooperation. Energy is partially lost during transfer, exposing a trade-off between energy cooperation and use of harvested energy for transmission. A multi-access relay model is considered and transmit power allocation and energy transfer policies that jointly maximize the sum-rate are found. It is shown that a class of power policies achieves the optimal sum-rate, allowing a separation of optimal energy transfer and optimal power allocation problems. The optimal energy transfer policy is shown to be an ordered node selection, where nodes with better energy transfer efficiency and worse channels transfer all their energy to the relay or other source nodes via the relay. For the special case of single source, the optimal policy requires the direction of energy transfer to remain unchanged unless either node depletes all of its energy. Overall, the findings provide the insight that cooperation of the source nodes by sharing energy with the relay node leads to them indirectly cooperating with each other, and that such cooperation can be carried out in a last-minute fashion.
\end{abstract}

\section{INTRODUCTION}

Energy harvesting enables wireless nodes to operate perpetually by using ambient energy, significantly extending network lifetime and decreasing maintenance costs [1]. In such networks, due to the intermittent nature of energy sources, some nodes may become energy deprived, calling for carefully designed energy management policies for data transmission. Recent advances in wireless energy transfer [2] bring the additional possibility of sharing energy, leading to the concept of energy cooperation to such wireless networks [3]. This paper is concerned with such a setting where a relay provides signal cooperation, and energy transfer to and from the relay provides energy cooperation.

Design and analysis of energy harvesting networks have recently been considered in a number of references, in particular in the context of obtaining transmission policies to amortize the available energy. A single user channel was considered in the studies that initiated this line of research in references [4], [5] for transmission completion time minimization with infinite battery transmitter, and for short-term throughput maximization with a finite battery transmitter, respectively. Extensions to fading, broadcast, multiple access, interference channels were considered in [6], [7], [8] and [9] respectively. Special cases of a two-hop channel with one energy harvesting source and one relay were studied in references [10], [11] with a half-duplex relay with one energy arrival and with two energy arrivals at the transmitter and relay respectively.

More recent developments in energy harvesting communications includes the possibility of energy transfer between nodes [3], [12], [13], [14]. Of particular relevance is reference [3] where the two-hop model with one source one relay is studied when both nodes are energy harvesting and the source can unidirectionally transfer energy to the relay. In this reference, the relay is assumed to buffer incoming data resulting in optimal policies identifiable in special cases only and rendering an analytical solutions challenging. Reference [12] considers the two-way and multiple access channels with unidirectional energy cooperation, proposing a two-dimensional directional water-filling algorithm with meters to obtain the optimal policy. Reference [13] considers the two-way channel for a binary energy exchange model.

In this work, we consider a network where a cooperating relay facilitates transmission from multiple sources to a destination, i.e., a multi-access relay channel. Each transmitter including the relay node harvests its energy to use for transmission, and has the option of transferring energy to another node in order for the overall sum-rate of the network to improve. We term this bi-directional energy cooperation. The ability of each transmitter transferring energy to the relay and receiving energy from the relay brings in the notion of implicit cooperation between the transmitters despite not having data cooperation links between them. Relevant to this set-up is a relay that does not store information from the sources, i.e., without a data buffer. In such a set-up, a relay with a healthy rate of energy harvests may opt to transfer some of its harvested energy to the sources less fortunate than itself, so as to be able to receive data to forward. We formulate and solve the problem of sumrate optimization in this general model by means of first proving that a class of feasible policies referred to as procrastinating policies, is sufficient to find the optimal sum-rate. With this observation, we show that the sum rate maximization problem can be decomposed into optimal energy transfer and optimal power allocation problems, subsequently solving them. While our solution is for the multi-source model, we also look into the special cases of the single source model, and the single source model with a forward energy transfer only, i.e., that 


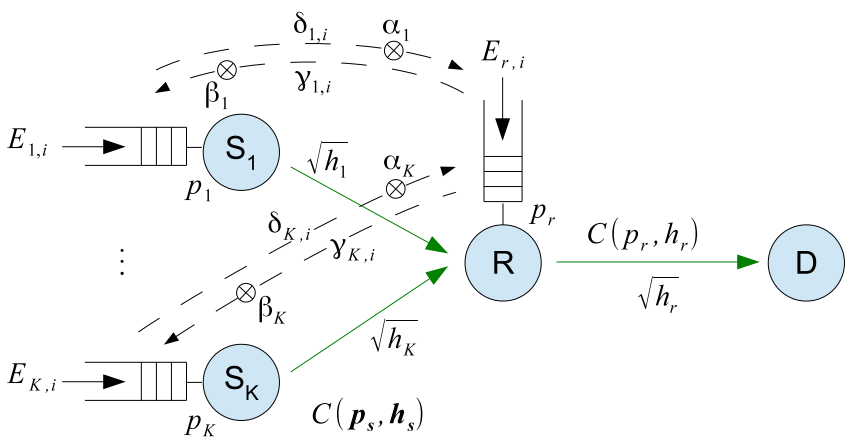

Fig. 1. Multiple source energy harvesting two-hop network model with twoway energy transfer.

of [3] without a data buffer at the relay, and provide the optimal policies. The differentiating contributions of this work with respect to previous work therefore can be summarized as follows:

- Introduction of a multi-source relay channel model with energy harvesting transmitters where the sources and the relay can cooperate by transferring energy to one another, while data cooperation is provided by the relay node that serve all the sources.

- Identification of a novel class of optimal energy cooperation policies wherein the nodes energy-cooperate only when the energy receiving node has immediate need to use it.

- Providing an analytical and clean description of the resulting jointly optimal energy cooperation and transmission power policies by a modified directional waterfilling algorithm.

\section{System Model And Problem Definition}

We consider a two-hop Gaussian relay network with $K$ energy harvesting sources and a relay node that can cooperate by sharing their energy, depicted in Figure 1. The source nodes $S_{k}, \quad k=1, \ldots, K$ have an infinite backlog of independent data. The relay node $R$, operating in full-duplex mode, decodes and forwards messages immediately to the destination node. This allows for a simpler relay design in practice and is necessary for delay-limited applications. We define ${ }^{1} C(\mathbf{p}, \mathbf{h})=$ $\frac{1}{2} \log \left(1+\mathbf{h}^{T} \mathbf{p}\right)$, where the vector $\mathbf{p}$ represents transmit powers and the vector $\mathbf{h}$ represents channel coefficients. We denote the transmit power of source nodes as $\mathbf{p}_{s}=\left[p_{1}, p_{2}, \ldots, p_{K}\right]^{T}$, the transmit power of the relay node as $p_{r}$, and the channel coefficients for the source-relay links and the relay-destination link as $\mathbf{h}_{s}=\left[h_{1}, h_{2}, \ldots, h_{K}\right]^{T}$ and $h_{r}$ respectively. The sum-rate achieved by decode-and-forward relaying is given by ${ }^{2}$

$$
R\left(\mathbf{p}_{s}, p_{r}\right)=\min \left\{C\left(\mathbf{p}_{s}, \mathbf{h}_{s}\right), C\left(p_{r}, h_{r}\right)\right\} .
$$

A time slotted model for energy harvests is considered with a slot length of $T$. At the beginning of the $i^{t h}$ time slot, the source nodes and the relay node harvest energy packets of size

\footnotetext{
${ }^{1} \mathbf{a}^{T}$ denotes the transpose of vector $\mathbf{a}$.

${ }^{2}$ The relay subtracts self interference from its received signal, thus achieving the Gaussian multiple access channel sum-capacity in the first hop.
}

$E_{k, i}, \quad k=1, \ldots, N$ and $E_{r, i}$ respectively. Furthermore, during the $i^{\text {th }}$ slot, each source node $S_{k}$ transfers energy to the relay at a rate $\delta_{k, i} \geq 0$, with an efficiency of $\alpha_{k} \leq 1$, while the relay node transfers energy at rate $\gamma_{k, i} \geq 0$ to source $S_{k}$ with an efficiency of $\beta_{k} \leq 1$. Consequently, a source node receives an energy of $\beta_{k} \gamma_{k, i} T$ in time slot $i$, while the relay receives $\sum_{k=1}^{K} \alpha_{k} \delta_{k, i} T$. It is assumed that for the duration of one time-slot, the energy harvest, transfer and consumption rates are constant. Hence, it is mathematically sufficient to formulate the energy constraints over total harvested, consumed and transferred energy in each time slot.

We denote the transmit powers of nodes $S_{k}$ and $R$ in time slot $i$ as $p_{k, i}$ and $p_{r, i}$ respectively. The energy available at each transmitting node at the end of the $i^{t h}$ time slot, i.e., prior to receiving $i+1^{\text {st }}$ energy arrival, is given by

$$
\begin{aligned}
E_{k, i}^{b a t} & =\sum_{j=1}^{i} E_{k, j}-\delta_{k, j} T+\beta_{k} \gamma_{k, j}-p_{k, j} T \\
E_{r, i}^{b a t} & =\sum_{j=1}^{i} E_{r, j}-\left(\sum_{k=1}^{K} \gamma_{k, j}+\alpha_{k} \delta_{k, j}\right) T-p_{r, j} T
\end{aligned}
$$

for sources $S_{k}$ and the relay respectively. The energy causality conditions for the first $N$ slots are expressed as

$$
E_{k, i}^{b a t} \geq 0, \quad E_{r, i}^{b a t} \geq 0, \quad k=1, \ldots, K, \quad i=1, \ldots, N .
$$

We consider the problem of maximizing the average sumrate within a deadline of $N$ time slots, through the choice of transmit powers $p_{k, i}, p_{r, i}$ and energy transfer rates $\delta_{k, i}$ and $\gamma_{k, i}$. This problem is formulated as

$$
\begin{aligned}
\max _{\mathbf{p}_{k}, \mathbf{p}_{r}, \boldsymbol{\delta}_{k}, \boldsymbol{\gamma}_{k}} & \frac{1}{N} \sum_{i=1}^{N} R\left(\mathbf{p}_{s, i}, p_{r, i}\right) \\
\text { s.t. } & \sum_{j=1}^{i} E_{k, j}-\left(\delta_{k, j}+\beta_{k} \gamma_{k, j}-p_{k, j}\right) T \geq 0 \\
& \sum_{j=1}^{i} E_{r, j}-\left(\sum_{k=1}^{K} \gamma_{k, j}+\alpha_{k} \delta_{k, j}\right) T-p_{r, j} T \geq 0 \\
& \mathbf{p}_{s, i} \geq 0, p_{r, i} \geq 0, \delta_{k, i} \geq 0, \gamma_{k, i} \geq 0 \\
& k=1, \ldots, K, i=1, \ldots, N
\end{aligned}
$$

where $\mathbf{p}_{k}, \mathbf{p}_{r}, \boldsymbol{\delta}_{k}$ and $\gamma_{k}$ are the vector representations of the transmit powers and energy transfer rates over $i=1, \ldots, N$, referred to as the power policy of the network in the sequel.

\section{Optimality of Procrastinating Power Policies}

For simple one-hop models with concave objectives and without energy transfer, it was shown in [4], [5] that the optimal power policy is unique. However, the two-hop model and the energy transfer capability in our model introduce new dimensions to the problem, resulting in the optimum solution not being unique in general. This can be readily verified by observing that energy transferred from an energy-rich $S_{k}$ to $R$ in slot $i$ can equivalently be transferred at a time prior to 
slot $i$. In order to simplify the problem, we first eliminate this freedom without affecting the optimality of the resulting policy by restricting our attention to a subset of policies.

Lemma 1: Define a procrastinating policy as a power policy comprised of $\mathbf{p}_{k}, \mathbf{p}_{r}, \boldsymbol{\delta}_{k}$ and $\gamma_{k}, k=1, \ldots, K$ satisfying

$$
\begin{aligned}
& p_{r, i} \geq \sum_{k=1}^{K} \alpha_{k} \delta_{k, i}-\gamma_{k, i}, \\
& p_{k, i} \geq \beta_{k} \gamma_{k, i}-\delta_{k, i}, \quad \delta_{k, i} \gamma_{k, i}=0
\end{aligned}
$$

for all $i=1, \ldots, N$ and $k=1, \ldots, K$. There exists at least one feasible procrastinating policy that solves the problem in (4).

Proof: We prove this statement by showing that for any feasible power policy, there exists a feasible procrastinating policy that achieves the same sum-rate. Observe that replacing $\delta_{k, i}$ and $\gamma_{k, i}$ with $\delta_{k, i}-\beta_{k} \gamma_{k, i}$ and 0 respectively when $\delta_{k, i} \geq$ $\gamma_{k, i}>0$, and replacing with 0 and $\gamma_{k, i}-\alpha_{k} \delta_{k, i}$ respectively when $\gamma_{k, i}>\delta_{k, i}>0$ does not reduce energy availability for any $i$. Hence, optimality of $\delta_{k, i} \gamma_{k, i}=0$ is readily shown. Next, assume that a policy $\left(\mathbf{p}_{k}, \mathbf{p}_{r}, \boldsymbol{\delta}_{k}, \boldsymbol{\gamma}_{k}\right)$ with $\delta_{k_{i}} \gamma_{k_{i}}=0$ is not procrastinating, in which case there exists at least one $k$ and $j$ for which $p_{r, j}<\sum_{k=1}^{K} \alpha_{k} \delta_{k, j}$ or $p_{k, j}<\beta_{k} \gamma_{k, j}$, leading to a nonzero portion of the transferred energy being stored in relay $R$ or source $S_{k}$. Choosing the smallest such $j$, we modify the policy by first substituting all nonzero $\delta_{k, j}$ with $\bar{\delta}_{k, j}=p_{r, j} / \alpha_{k}$ for the former case, or $\bar{\gamma}_{k, j}=p_{k, j} / \beta_{k}$ for the latter case, while keeping all other variables the same. If $j=N$, or if all constraints for $i>j$ are still satisfied, the modified policy is feasible. If any constraint is violated in slot $\ell>j$, we set $\bar{\delta}_{k, \ell}=\delta_{k, \ell}+\delta_{k, j}-p_{r, j} / \alpha_{k}$ for the former case, or $\bar{\gamma}_{k, \ell}=\gamma_{k, \ell}+\gamma_{k, j}-p_{k, j} / \beta_{k}$ for the latter case, which ensures that all constraints for $i \geq \ell$ are identical to those for the original policy and are therefore satisfied. Notice that this modification does not change $\mathbf{p}_{k}$ or $\mathbf{p}_{r}$, and thus achieves the same sum-rate, while the smallest time-slot $i$ that prevents the policy from being procrastinating is strictly increased. By repeatedly modifying the policy as such, a procrastinating policy which has the same sum-rate can be found for any non-procrastinating policy.

The set of procrastinating policies are called as such since no energy is transferred ahead of time with the intention of storage at the receiving side. In other words, given a fixed transmit power policy $\left(\mathbf{p}_{k}, \mathbf{p}_{r}\right)$, any energy transfer that is due for the feasibility of $\left(\mathbf{p}_{k}, \mathbf{p}_{r}\right)$ is delayed until it is immediately needed for transmission at the receiving node. Lemma 1 proves that an optimal policy can be found among procrastinating policies. We next use this observation to prove the separation of power allocation and energy transfer problems.

\section{Optimal Policy}

\section{A. Problem Decomposition}

We rewrite the problem in (4) in terms of

$$
\begin{aligned}
& \bar{p}_{r, i}=p_{r, i}+\sum_{k=1}^{K} \gamma_{k, i}-\alpha_{k} \delta_{k, i} \\
& \bar{p}_{k, i}=p_{k, i}+\left(\delta_{k, i}-\beta_{k} \gamma_{k, i}\right), \quad k=1, \ldots, K .
\end{aligned}
$$

Here, $\bar{p}_{k, i}$ and $\bar{p}_{r, i}$ can be interpreted as the powers drawn from the batteries of nodes $S_{k}$ and $R$ respectively for transmission or transfer in the $i^{\text {th }}$ time slot. A procrastinating policy satisfies $\bar{p}_{r, i} \geq 0$ and $\bar{p}_{k, i} \geq 0$ for all $i$ and $k$. The nonnegativity of $p_{k, i}$ and $p_{r, i}$ can be expressed through $\bar{p}_{k, i} \geq \delta_{k, i}$, and $\bar{p}_{r, i} \geq \sum_{k=1}^{K} \gamma_{k, i}$ respectively. The problem restricted to procrastinating policies, ignoring the constant term $1 / N$, can be rewritten as

$$
\begin{aligned}
& \max _{\substack{\overline{\mathbf{p}}_{\mathbf{s}}, \overline{\mathbf{p}}_{\mathbf{r}}, \boldsymbol{\delta}_{k}, \boldsymbol{\gamma}_{k}}} \sum_{i=1}^{N} R\left(\left[\bar{p}_{k, i}+\beta_{k} \gamma_{k, i}-\delta_{k, i}\right], \bar{p}_{r, i}+\sum_{k=1}^{K} \alpha_{k} \delta_{k, i}-\gamma_{k, i}\right) \\
& \text { s.t. } \sum_{j=1}^{i} E_{r, j}-\bar{p}_{r, j} T \geq 0, \quad \sum_{j=1}^{i} E_{k, j}-\bar{p}_{k, j} T \geq 0 \\
& \bar{p}_{k, i} \geq \delta_{k, i}, \quad \bar{p}_{r, i} \geq \sum_{k=1}^{K} \gamma_{k, i}, \quad \delta_{k, i}, \gamma_{k, i} \geq 0 \\
& k=1, \ldots, K, \quad i=1, \ldots, N,
\end{aligned}
$$

where we denote $\mathbf{p}_{s, i}=\left[p_{k, i}\right]$. Notice that the energy causality constraints in (9b) no longer depend on $\delta_{k}$ or $\gamma_{k}$, and each summation term in (9a) depends only on the corresponding $\delta_{k, i}$ and $\gamma_{k, i}$. Hence, the problem can be decomposed as

$$
\begin{aligned}
\max _{\overline{\mathbf{p}}_{s}, \overline{\mathbf{p}}_{r}} & \sum_{i=1}^{N} \max _{\substack{0 \leq \delta_{k, i} \leq \bar{p}_{k, i} \\
0 \leq \sum_{k=1}^{K} \gamma_{k, i} \leq \bar{p}_{r, i}}} R\left(\mathbf{p}_{s, i}, p_{r, i}\right) \\
\text { s.t. } & (9 b), \quad \bar{p}_{s, i} \geq 0, \quad \bar{p}_{r, i} \geq 0, i=1, \ldots, N
\end{aligned}
$$

and the inner maximization can be solved separately for the optimal $\delta_{k, i}$ and $\gamma_{k, i}$ given any $\bar{p}_{k, i}$ and $\bar{p}_{r, i}$.

\section{B. Optimal Energy Transfer}

For the rate function given in (1), the inner objective of (10a) can be shown to be maximized when

$$
\sum_{k=1}^{K} h_{k}\left(\bar{p}_{k, i}+\beta_{k} \gamma_{k, i}^{*}-\delta_{k, i}^{*}\right)=h_{r}\left(\bar{p}_{r, i}+\sum_{k=1}^{K} \alpha_{k} \delta_{k, i}^{*}-\gamma_{k, i}^{*}\right)
$$

while either side of the equality in (11) can be considered as the new objective. This is a linear problem, and its solution can be evaluated as follows: First, source nodes $k \in\{1, \ldots, K\}$ satisfying $h_{k} / \alpha_{k}<\beta_{m} h_{m}$ for any $m \in\{1, \ldots, K\}$ are found, in which case $S_{k}$ transfers all of its energy to the relay by setting $\delta_{k, i}=\bar{p}_{k, i}$. This energy is subsequently forwarded to the source node $S_{m}$ with the largest $\beta_{m} h_{m}$. Next, both sides of the equality in (11) are evaluated. If the LHS is greater, each source node $S_{k}$ is assigned the respective transfer priority $\pi_{k, \delta}=\alpha_{k} / h_{k}$ and transmit priority $\pi_{k, \gamma}=1 / \beta_{k} h_{k}$. The nodes are ordered in terms of both priorities, and starting from the node with the highest priority, energy transfer is performed if feasible until the equality in (11) is satisfied. In case of transfer priority, $\delta_{k, i}$ is increased, whereas in the case of transmit priority, $\gamma_{k, i}$ is decreased if feasible. Conversely, if the RHS is greater, the source nodes $S_{k}$ are assigned transmit priorities $\pi_{k, r}=\beta_{k} h_{k}$, and $\gamma_{k, i}$ for only the highest priority node is increased until the equality is achieved. Notice that this is an ordered node 
selection; when transferring energy to the relay, nodes with good transfer efficiency and bad channels are chosen, while when transferring energy to a source node, the node with a good transfer efficiency and a good channel is preferred.

\section{Optimal Power Allocation}

Once the energy transfer problem is solved, the rate achieved can be expressed in terms of consumed powers $\bar{p}_{k, i}$ and $\bar{p}_{r, i}$ alone. Denoting this function as $\bar{R}\left(\overline{\mathbf{p}}_{s, i}, \bar{p}_{r, i}\right)$, the remaining power allocation problem can be expressed as

$$
\begin{aligned}
\max _{\overline{\mathbf{p}}_{s}, \overline{\mathbf{p}}_{r}} & \sum_{i=1}^{N} \bar{R}\left(\overline{\mathbf{p}}_{s, i}, \bar{p}_{r, i}\right), \\
\text { s.t. } & (9 b), \overline{\mathbf{p}}_{s, i} \geq 0, \bar{p}_{r, i} \geq 0, i=1, \ldots, N .
\end{aligned}
$$

The objective in (15a) is jointly concave in $\bar{p}_{s, i}$ and $\bar{p}_{r, i}$ since $R\left(\mathbf{p}_{s, i}, p_{r, i}\right)$ is concave and $\bar{R}$ is the solution of a linear problem. Hence, (15) is a convex problem, and due to the constraints being separable in $\bar{p}_{k, i}$ and $\bar{p}_{r, i}$, it can be solved using block coordinate descent [15] among $\bar{p}_{k, i}$ and $\bar{p}_{r, i}$.

\section{Special Case: Single Source}

For clarity of exposition, we now focus on a two-hop network with a single source node, i.e., $K=1$, and find the optimal energy transfer and power allocation policies. Denoting the allocated power at the source as $p_{s, i}$ and the consumed power at the source as $\bar{p}_{s, i}$, and dropping the subscripts $k$ where applicable, (11) reduces to

$$
h_{s}\left(\bar{p}_{s, i}+\beta \gamma_{i}^{*}-\delta_{i}^{*}\right)=h_{r}\left(\bar{p}_{r, i}+\alpha \delta_{i}^{*}-\gamma_{i}^{*}\right)
$$

and a priority ordering is not necessary due to $K=1$. Thus, the optimal energy transfer rates $\delta_{i}^{*}, \gamma_{i}^{*}$ can be found by comparing the two sides of the equality in (13), and are given by

$$
\delta_{i}^{*}=\left[\frac{h_{s} \bar{p}_{s, i}-h_{r} \bar{p}_{r, i}}{\alpha h_{r}+h_{s}}\right]^{+}, \gamma_{i}^{*}=\left[\frac{h_{r} \bar{p}_{r, i}-h_{s} \bar{p}_{s, i}}{h_{r}+\beta h_{s}}\right]^{+},
$$

Note that the sign of $h_{s} \bar{p}_{s, i}-h_{r} \bar{p}_{r, i}$ is the critical factor in determining which transmitter will be transferring energy. In the sequel, we refer to the node with larger $h_{j} \bar{p}_{j, i}, j \in\{s, r\}$, as the generous transmitter for transferring energy to the other node. Substituting this result in (15), the optimal policy can be found as the solution to

$$
\begin{aligned}
& \max _{\overline{\mathbf{p}}_{s}, \overline{\mathbf{p}}_{r}} \sum_{i=1}^{N} R\left(\bar{p}_{s, i}+\beta \gamma_{i}^{*}-\delta_{i}^{*}, \bar{p}_{r, i}+\alpha \delta_{i}^{*}-\gamma_{i}^{*}\right), \\
& \text { s.t. }(9 b), \bar{p}_{s, i} \geq 0, \bar{p}_{r, i} \geq 0, i=1, \ldots, N .
\end{aligned}
$$

which can be solved using a block coordinate descent algorithm as discussed in Section IV-C. We refer to the iterations optimizing over $\bar{p}_{k, i}$ and $\bar{p}_{r, i}$ as the source and relay iterations respectively. The problem solved by each iteration is

$$
\begin{aligned}
\max _{\overline{\mathbf{p}}_{j}} & \sum_{i=1}^{N} \log \left(1+h_{s} h_{r} \min \left\{\frac{\bar{p}_{s, i}+\beta \bar{p}_{r, i}}{\beta h_{s}+h_{r}}, \frac{\alpha \bar{p}_{s, i}+\bar{p}_{r, i}}{h_{s}+\alpha h_{r}}\right\}\right), \\
\text { s.t. } & \sum_{k=1}^{i} E_{j, k}-\bar{p}_{j, k} T \geq 0, \bar{p}_{j, i} \geq 0, i=1, \ldots, N .
\end{aligned}
$$

where $j=s$ gives the source iteration and $j=r$ gives the relay iteration. Observe that (16) is the short-term throughput maximization problem for a single transmitter without energy transfer, but with a different rate function. Therefore, we find the solution to each iteration using a generalized directional water-filling algorithm [8], [9]. This algorithm differs from that in [6] in using generalized water levels found as the inverse of the partial derivative of the rate function. The generalized water levels are given by

$$
v_{s, i}= \begin{cases}\bar{p}_{s, i}+\beta \bar{p}_{r, i}+\frac{\beta h_{s}+h_{r}}{h_{s} h_{r}} & h_{s} \bar{p}_{s, i}<h_{r} \bar{p}_{r, i} \\ \bar{p}_{s, i}+\frac{\bar{p}_{r, i}}{\alpha}+\frac{\alpha h_{r}+h_{s}}{h_{s} h_{r} \alpha} & h_{s} \bar{p}_{s, i} \geq h_{r} \bar{p}_{r, i}\end{cases}
$$

for the source iterations and

$$
v_{r, i}= \begin{cases}\bar{p}_{r, i}+\frac{\bar{p}_{s, i}}{\beta}+\frac{\beta h_{s}+h_{r}}{h_{s} h_{r} \beta} & h_{s} \bar{p}_{s, i}<h_{r} \bar{p}_{r, i} \\ \bar{p}_{r, i}+\alpha \bar{p}_{s, i}+\frac{\alpha h_{r}+h_{s}}{h_{s} h_{r}} & h_{s} \bar{p}_{s, i} \geq h_{r} \bar{p}_{r, i}\end{cases}
$$

for the relay iterations. Notice that the water level expressions depend on which node is generous. The generalized water levels in (17) and (18) point to a conventional water-filling interpretation due to being linear in transmit powers. The base levels are affected by the transmit power of the other user as well as a constant term. Since the base levels are fixed within each iteration, a directional water-filling procedure [6] can be used to find the optimal power allocation. Finally, transmit powers can be calculated by finding the optimal transfer rates $\delta_{i}$ and $\gamma_{i}$ using (14).

We remark that $v_{s, i}=\beta v_{r, i}$ for $h_{s} \bar{p}_{s, i}<h_{r} \bar{p}_{r, i}$, and $v_{s, i}=v_{r, i} / \alpha$ for $h_{s} \bar{p}_{s, i} \geq h_{r} \bar{p}_{r, i}$, implying that water levels are linearly related based on which transmitter is generous in that time slot. An immediate consequence is that for the water levels of two neighboring time slots to be equal for both iterations, the same node must be generous in both time slots. Since water level may only change at empty battery instances in a directional water-filling algorithm, an insightful property of the optimal policy arises: the generous node, and hence the direction of energy transfer, does not change unless one of the nodes is energy deprived.

\section{Special CASE: Unidirectional EnERgy Transfer}

Consider the special case with a single source node and $\beta=$ 0 , i.e., the unidirectional energy cooperation model studied in [3]. For this case, we can set $\gamma_{i}=0$ for all $i$, and therefore the set of procrastinating policies only need to satisfy $p_{r, i} \geq \alpha \delta_{i}$. The generalized water levels from (17) and (18) become

$$
\begin{aligned}
& v_{s, i}= \begin{cases}\bar{p}_{s, i}+\frac{1}{h_{s}} & h_{s} \bar{p}_{s, i}<h_{r} \bar{p}_{r, i} \\
\bar{p}_{s, i}+\frac{\bar{p}_{r, i}}{\alpha}+\frac{\alpha h_{r}+h_{s}}{h_{s} h_{r} \alpha} & h_{s} \bar{p}_{s, i} \geq h_{r} \bar{p}_{r, i}\end{cases} \\
& v_{r, i}= \begin{cases}\infty & h_{s} \bar{p}_{s, i}<h_{r} \bar{p}_{r, i} \\
\bar{p}_{r, i}+\alpha \bar{p}_{s, i}+\frac{\alpha h_{r}+h_{s}}{h_{s} h_{r}} & h_{s} \bar{p}_{s, i} \geq h_{r} \bar{p}_{r, i}\end{cases}
\end{aligned}
$$

Here, the infinite water level in (20) implies that the relay never chooses a power satisfying $h_{s} \bar{p}_{s, i}<h_{r} \bar{p}_{r, i}$, since any power in excess of $h_{s} p_{s, i} / h_{r}$ does not contribute to the objective. Also note that $v_{r, i}=\alpha v_{s, i}$ for $h_{s} \bar{p}_{s, i} \geq h_{r} \bar{p}_{r, i}$, implying that directional water-filling requirements on the relay iteration 

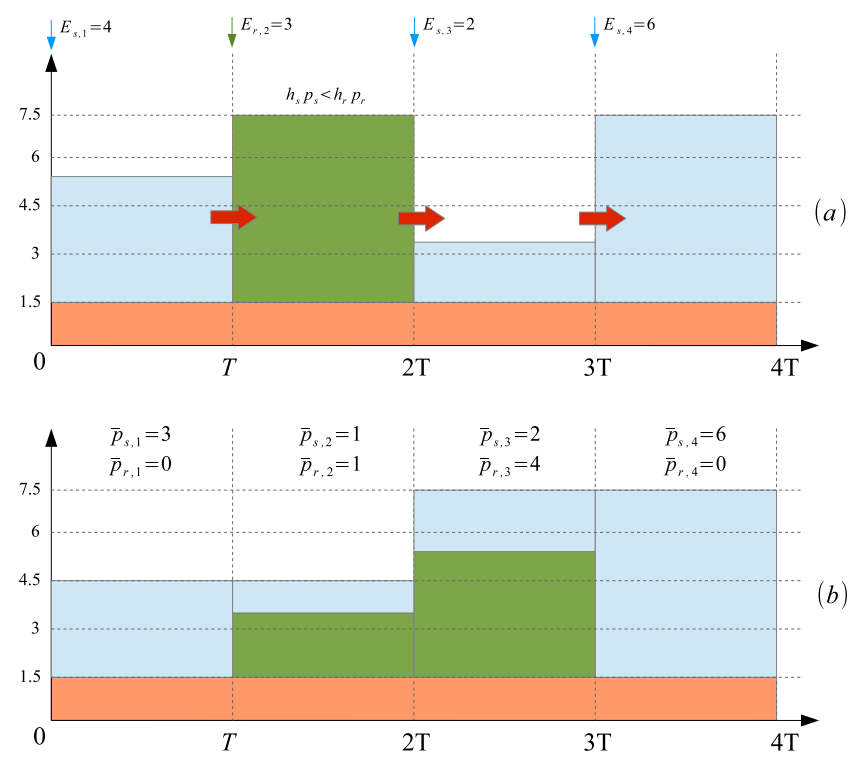

Fig. 2. Directional water-filling (a) initial levels and (b) solution for an example setting with $h_{s}=h_{r}=1, T=1, N=4, \alpha=0.5, E_{s}=[4,0,2,6]$ and $E_{r}=[0,3,0,0]$. The blue, green and orange areas correspond to source consumption $\bar{p}_{s}$, relay consumption $\bar{p}_{r}$ and the constant water level term in (17) respectively.

are satisfied as long as directional water-filling requirements on the source iteration are satisfied and $h_{s} \bar{p}_{s, i} \geq h_{r} \bar{p}_{r, i}$ is met. Hence, both iterations can be evaluated in parallel by a single water-filling algorithm, with water levels given by

$$
v_{i}=\bar{p}_{s, i}+\frac{\bar{p}_{r, i}}{\alpha}+\frac{\alpha h_{r}+h_{s}}{h_{s} h_{r} \alpha}
$$

while ensuring that $h_{s} \bar{p}_{s, i} \geq h_{r} \bar{p}_{r, i}$ is satisfied in each epoch. The algorithm can be interpreted as waterfilling with two non-mixing fluids corresponding to $\bar{p}_{s}$ and $\bar{p}_{r} / \alpha$, with each fluid flowing only in forward direction and the total water level obeying the directional water-filling restrictions such as water level increasing only when a node is out of energy. An example demonstrating this algorithm is given in Figure 2 with $h_{s}=h_{r}=1, T=1, N=4$ and $\alpha=0.5$, for $E_{s}=[4,0,2,6]$ and $E_{r}=[0,3,0,0]$. Notice that although water level could be stabilized at 5.5 in the second time slot, relay energy is moved to the third slot until $h_{s} p_{s, i}<h_{r} p_{r, i}$ is satisfied.

\section{CONCLUSION}

In this paper, the energy harvesting multiple access relay channel with bidirectional energy transfer is studied. The sumrate maximization problem is solved. It is shown that a subset of feasible policies, termed procrastinating policies, includes at least one power policy that yields the globally optimum sumrate. With this observation, energy transfer and transmission energy expenditure problems can be separated, rendering an analytically tractable optimal policy. The solution to the optimal energy transfer problem is shown to be an ordered node selection, where source nodes are prioritized over the strength of their wireless and energy transfer channels. The optimal power consumption problem is then solved using an iterative algorithm, which reduces to a directional water-filling algorithm when the number of sources is 1 . The special case with unidirectional energy transfer is revisited, and the directional water-filling algorithm is shown to solve the respective problem without any iterations.

It is also recently observed that the class of procrastinating policies can lead to optimal sum-rate in one-hop models [16]. Future directions include considering different network models with energy transfer, and considerations on finite energy storage and limited energy cooperation.

\section{ACKNOWLEDGEMENTS}

The authors would like to thank Prof. Sennur Ulukus of UMD for discussions and constructive comments on this paper.

\section{REFERENCES}

[1] X. Jiang, J. Polastre, and D. Culler, "Perpetual environmentally powered sensor networks," in Fourth International Symposium on Information Processing in Sensor Networks,IPSN, 2005, pp. 463-468.

[2] A. Karalis, J. Joannopoulos, and M. Soljačić, "Efficient wireless nonradiative mid-range energy transfer," Annals of Physics, vol. 323, no. 1, pp. 34-48, 2008.

[3] B. Gurakan, O. Ozel, J. Yang, and S. Ulukus, "Energy cooperation in energy harvesting wireless communications," in Proceedings of IEEE International Symposium on Information Theory, ISIT, Jul. 2012, pp. 965969.

[4] J. Yang and S. Ulukus, "Optimal packet scheduling in an energy harvesting communication system," IEEE Transactions on Communications, vol. 60 , no. 1, pp. 220-230, 2012.

[5] K. Tutuncuoglu and A. Yener, "Optimum transmission policies for battery limited energy harvesting nodes," IEEE Transactions on Wireless Communications, vol. 11, no. 3, pp. 1180-1189, Mar. 2012.

[6] O. Ozel, K. Tutuncuoglu, J. Yang, S. Ulukus, and A. Yener, "Transmission with energy harvesting nodes in fading wireless channels: Optimal policies," IEEE Journal on Selected Areas in Communications, vol. 29, no. 8, pp. $1732-1743$, Sep. 2011.

[7] J. Yang, O. Ozel, and S. Ulukus, "Broadcasting with an energy harvesting rechargeable transmitter," IEEE Transactions on Wireless Communications, vol. 11, no. 2, pp. 571-583, 2012.

[8] J. Yang and S. Ulukus, "Optimal packet scheduling in a multiple access channel with energy harvesting transmitters," Communications and Networks, Journal of, vol. 14, no. 2, pp. 140-150, 2012.

[9] K. Tutuncuoglu and A. Yener, "Sum-rate optimal power policies for energy harvesting transmitters in an interference channel," JCN Special Issue on Energy Harvesting in Wireless Networks, vol. 14, no. 2, pp. 151-161, April 2012.

[10] D. Gunduz and B. Devillers, "Two-hop communication with energy harvesting," in Proceedings of the 4th International Workshop on Computational Advances in Multi-Sensor Adaptive Processing, (CAMSAP), Dec. 2011.

[11] O. Orhan and E. Erkip, "Optimal transmission policies for energy harvesting two-hop networks," in 46th Annual Conference on Information Sciences and Systems (CISS). IEEE, 2012, pp. 1-6.

[12] B. Gurakan, O. Ozel, J. Yang, and S. Ulukus, "Two-way and multipleaccess energy harvesting systems with energy cooperation," in 46th Asilomar Conference on Signals, Systems and Computers, Pacific Grove, CA, Nov. 2012.

[13] P. Popovski, A. Fouladgar, and O. Simeone, "Interactive joint transfer of energy and information," submitted to IEEE Transactions on Communications, 2012, available at arXiv:1209.6367.

[14] Y.-K. Chia, S. Sun, and R. Zhang, "Energy cooperation in cellular networks with renewable powered base stations," arXiv preprint arXiv:1301.4786, 2013.

[15] D. Bertsekas, Nonlinear programming. Athena Scientific, 1999.

[16] K. Tutuncuoglu and A. Yener, "Multiple access and two-way channels with energy harvesting and bidirectional energy cooperation," in Proceedings of the 2013 Information Theory and Applications Workshop, ITA'13, Feb. 2013. 